Preprint typeset in JHEP style. - HYPER VERSION

hep-ph/0109078

SLAC-PUB-8976

$\mathrm{UCLA} / 01 / \mathrm{TEP} / 16$

September, 2001

\title{
Two-Loop Amplitudes for Gluon Fusion into Two Photons
}

\author{
Z. Bern,* A. De Freitas* \\ Department of Physics and Astronomy \\ UCLA, Los Angeles, CA 90095-1547 \\ L. Dixon $^{\dagger}$ \\ Stanford Linear Accelerator Center \\ Stanford University \\ Stanford, CA 94309
}

\begin{abstract}
We present the two-loop matrix elements for the scattering of two gluons into two photons in QCD. These matrix elements will enter into improved estimates of the QCD background to Higgs production at the LHC when the Higgs decays into two photons. The photon mode is especially important if $M_{H}<140 \mathrm{GeV}$. We also give the amplitudes for the crossed process, $g \gamma \rightarrow g \gamma$.
\end{abstract}

KEYwords: two-loop, QCD, quantum chromodynamics, Higgs, photons.

Submitted to JHEP

\footnotetext{
*Research supported by the US Department of Energy under grant DE-FG03-91ER40662.

${ }^{\dagger}$ Research supported by the US Department of Energy under grant DE-AC03-76SF00515.
} 


\section{Introduction}

One of the most pressing problems in particle physics today is to determine the nature of electroweak symmetry breaking. Experiments in the coming decade at the Fermilab Tevatron and the CERN Large Hadron Collider (LHC) will address this problem, in particular by searching for one or more Higgs bosons. There are good reasons to think that at least one such particle will be fairly light. In the Standard Model the Higgs boson is constrained to be light by its influence on precision electroweak measurements, $m_{H} \lesssim 205-230 \mathrm{GeV}$ at $95 \% \mathrm{CL}$ [1]. In the Minimal Supersymmetric Standard Model, the lightest Higgs boson is predicted to have a mass below about $135 \mathrm{GeV}$ [2]; over much of the parameter space it has properties reasonably similar to the Standard Model Higgs boson. Finally, there are hints from LEP at $2.9 \sigma$ significance of a Higgs boson at $m_{H}=115 \mathrm{GeV}$ [3].

To aid in the search for the Higgs boson, it is useful to have a detailed understanding of the Standard Model backgrounds. At the LHC the most important mode for discovering a Higgs boson with $m_{H}<140 \mathrm{GeV}$ is via its decay into two photons [4, 5]. The irreducible two-photon background due to QCD, $p p \rightarrow \gamma \gamma X$, will be determined experimentally at the LHC, so the Higgs search can proceed even with imprecise theoretical knowledge of it. Nevertheless, it is still of interest to have robust theoretical predictions prior to the experiments to help optimize Higgs search strategies. In this paper we provide the two-loop matrix elements for $g g \rightarrow \gamma \gamma$ with massless quarks in the loop, which form a central ingredient in an improved prediction of the irreducible background.

The process $p p \rightarrow \gamma \gamma X$ proceeds at lowest order via the partonic subprocess $q \bar{q} \rightarrow \gamma \gamma$, which is independent of $\alpha_{s}$. The next-to-leading-order (NLO) corrections to this subprocess have been incorporated into a number of Monte Carlo programs [6]. However, at the LHC, due to the high gluon luminosity, formally higher order corrections involving gluon initial states are sizable. The contribution from the lowest order $g g \rightarrow \gamma \gamma$ amplitudes (from one-loop box diagrams), though of order $\alpha_{s}^{2}$, is very comparable in size to the $q \bar{q} \rightarrow \gamma \gamma$ contribution [5, 7, 6]. Thus a calculation of the $g g \rightarrow \gamma \gamma$ subprocess at its next-to-leading-order (which is formally $\mathrm{N}^{3} \mathrm{LO}$ as far as the whole process $p p \rightarrow \gamma \gamma X$ is concerned), should lead to a significant reduction in the uncertainty on the total cross section. A calculation of the background, incorporating the two-loop matrix elements presented in this paper, will appear in a forthcoming publication [8]. In the range of di-photon invariant masses relevant for the Higgs search, 90-150 GeV, quark masses may be neglected. The $u, d, s, c$ and $b$ quark masses are all much less than the scale of the process, while the top quark contribution is negligible until the invariant mass approaches $2 m_{t} \approx 350 \mathrm{GeV}$. 
The subprocess $g \gamma \rightarrow g \gamma$ does not possess quite the same phenomenological significance as $g g \rightarrow \gamma \gamma$. However, it does receive a large power-enhanced correction at two loops in the forward scattering limit, due the exchange of two gluons, which represents the beginning of the Reggeization of this amplitude. The amplitudes for $g g \rightarrow \gamma \gamma$ and $g \gamma \rightarrow g \gamma$ are of course related by crossing symmetry, but writing the full two-loop amplitude in a crossing-symmetric form would be more cumbersome, so we shall present each case separately.

Calculating two-loop four-point amplitudes involving more than a single kinematic variable is a relatively new art. The first calculations of this type in gauge theory were for the special cases of gluon-gluon scattering with maximal supersymmetry [9] and maximal helicity violation in QCD [10]. More recently, more general calculations of interferences of two-loop amplitudes with tree amplitudes in QED and in QCD have appeared. In QED the interferences for $e^{+} e^{-} \rightarrow \mu^{+} \mu^{-}$and $e^{+} e^{-} \rightarrow e^{+} e^{-}$[11] have been computed. In a tour de force calculation, Anastasiou, Glover, Oleari, and TejedaYeomans have provided the interferences for all QCD $2 \rightarrow 2$ parton processes [12, 13].

The two-loop Feynman diagrams for the $g g \rightarrow \gamma \gamma$ matrix elements presented in this paper are similar to those required for gluon-gluon scattering [13], $g g \rightarrow g g$, except that many of the non-Abelian diagrams are not present. In the $g g \rightarrow \gamma \gamma$ case, the tree amplitudes vanish and the one-loop amplitudes give the leading order contributions. Thus the next-to-leading order contributions to this process require a different interference, of two-loop amplitudes with one-loop amplitudes. Instead of evaluating this interference directly, we have computed the two-loop $g g \rightarrow \gamma \gamma$ amplitudes in a helicity basis. We employed a unitarity- or cut-based technique [14, 9, 10] to generate the required loop momentum integrals. These integrals were then evaluated using recently developed techniques.

Two important technical breakthroughs which have provided the required integrals for general $2 \rightarrow 2$ scattering in the massless case are the calculations of the dimensionally regularized scalar double box integrals with planar [15] and non-planar [16] topologies and all external legs massless, and the development of reduction algorithms for the same types of integrals with loop momenta in the numerator (tensor integrals) [17, 18, 19]. Related integrals which also arise in the reduction procedure have been computed in refs. 20]. Taken together, these results are sufficient to compute all loop integrals required for $2 \rightarrow 2$ massless scattering amplitudes at two loops.

A helpful development for performing explicit two-loop calculations is a general formula due to Catani for the infrared divergence appearing in any two-loop QCD amplitude [21]. By appropriately adjusting group theory factors, it is straightforward to apply Catani's QCD formula to amplitudes including external photons. Because of the vanishing of the tree amplitudes, the infrared divergences for $g g \rightarrow \gamma \gamma$ and $g \gamma \rightarrow g \gamma$ 
are much tamer than those of a typical QCD process at two loops. Indeed, Catani's formula collapses to a form previously derived for one loop amplitudes [22, 23]. We present the amplitudes in terms of Catani's formula for the infrared divergences, plus finite remainders for each independent helicity configuration.

In the physical next-to-leading-order correction to the $g g \rightarrow \gamma \gamma$ subprocess of $p p \rightarrow \gamma \gamma X$, the infrared divergences from the two-loop amplitude cancel against those arising from phase-space integration of the (square of) the one-loop amplitudes for $g g \rightarrow \gamma \gamma g$ [24, 25], after factorizing the initial-state collinear singularities. Both these "virtual" and "real" divergences are effectively the same as encountered at one loop, so standard one-loop formalisms [26] can be employed to obtain the cross section [8].

This paper is organized as follows. In section 2 we review the infrared structure of the two-loop $g g \rightarrow \gamma \gamma$ and $g \gamma \rightarrow g \gamma$ amplitudes. The corresponding one-loop amplitudes, which appear in the formula for the infrared divergences of the two-loop amplitudes, are presented in section 3 . In sections 1 and 5 we present analytic results for the finite remainders of the two-loop $g g \rightarrow \gamma \gamma$ and $g \gamma \rightarrow g \gamma$ helicity amplitudes, respectively. In section 6 we discuss the checks we performed on our results. Finally, in section 7 we present our conclusions.

\section{Review of infrared structure}

In presenting the amplitudes it is convenient to separate the infrared divergent parts from the finite parts. Dimensionally regulated two-loop amplitudes for four massless particles in $D=4-2 \epsilon$ dimensions generically contain poles in $\epsilon$ up to $1 / \epsilon^{4}$. Catani has presented a general formula for the structure of infrared divergences of any QCD amplitude [21]. With minor adjustments, Catani's formula is also valid for the case of mixed amplitudes of QED and QCD. We shall therefore adopt his notation in presenting our results.

We work with ultraviolet renormalized amplitudes, and employ the $\overline{\mathrm{MS}}$ running coupling for QCD, $\alpha_{s}\left(\mu^{2}\right)$. Since the tree amplitudes for the process under consideration vanish, for purposes of renormalization we only need the one-loop relation between the bare coupling $\alpha_{s}^{u}$ and renormalized coupling $\alpha_{s}\left(\mu^{2}\right)$,

$$
\alpha_{s}^{u} \mu_{0}^{2 \epsilon} S_{\epsilon}=\alpha_{s}\left(\mu^{2}\right) \mu^{2 \epsilon}\left[1-\alpha_{s}\left(\mu^{2}\right) \frac{\beta_{0}}{\epsilon}+\mathcal{O}\left(\alpha_{s}^{2}\left(\mu^{2}\right)\right)\right]
$$

where $S_{\epsilon}=\exp [\epsilon(\ln 4 \pi+\psi(1))]$ and $\gamma=-\psi(1)=0.5772 \ldots$ is Euler's constant. The coefficient appearing in the QCD beta function is

$$
\beta_{0}=\frac{11 C_{A}-4 T_{R} N_{f}}{12 \pi},
$$


where $N_{f}$ is the number of light (massless) quarks, $C_{A}=N$ for $S U(N)$ and $T_{R}=1 / 2$ for fundamental representation Dirac fermions.

The renormalized QCD corrections to the $g g \rightarrow \gamma \gamma$ amplitude discussed in this paper may be expanded as

$$
\begin{aligned}
\mathcal{M}_{g g \rightarrow \gamma \gamma}\left(\alpha_{s}\left(\mu^{2}\right), \alpha, \mu^{2} ;\{p\}\right)=4 \pi \alpha & {\left[\frac{\alpha_{s}\left(\mu^{2}\right)}{2 \pi} \mathcal{M}_{g g \rightarrow \gamma \gamma}^{(1)}\left(\mu^{2} ;\{p\}\right)\right.} \\
& \left.+\left(\frac{\alpha_{s}\left(\mu^{2}\right)}{2 \pi}\right)^{2} \mathcal{M}_{g g \rightarrow \gamma \gamma}^{(2)}\left(\mu^{2} ;\{p\}\right)+\mathcal{O}\left(\alpha_{s}^{3}\left(\mu^{2}\right)\right)\right] .
\end{aligned}
$$

where $\mathcal{M}_{g g \rightarrow \gamma \gamma}^{(L)}\left(\mu^{2} ;\{p\}\right)$ is the $L$ th loop contribution. Since there is no direct coupling between gluons and photons, the expansion starts at one loop.

The QCD $\overline{\mathrm{MS}}$ counterterm which is subtracted from the bare two-loop amplitude to obtain the renormalized $\mathcal{M}_{g g \rightarrow \gamma \gamma}^{(2)}\left(\mu^{2} ;\{p\}\right)$ is (cf. eq. (2.1))

$$
\text { C.T. }=\frac{11 N-2 N_{f}}{6} \frac{1}{\epsilon} \mathcal{M}_{g g \rightarrow \gamma \gamma}^{(1)}\left(\mu^{2} ;\{p\}\right) \text {. }
$$

The relative simplicity of the ultraviolet subtraction term is due to the vanishing of the tree-level amplitudes.

There is also a QED divergence associated with the diagrams in Figure 1. In dimensional regularization with massless fermions, these diagrams would vanish by virtue of containing a scale free integral. This vanishing represents a cancellation between infrared and ultraviolet divergences. In QCD with $\overline{\mathrm{MS}}$ renormalization such diagrams (with the bubble on a gluon leg) are thus taken to vanish. However, if one renormalizes QED in the conventional on-shell scheme, to avoid the infrared divergences one should retain the fermion masses in the external bubbles. Now the bubble integral is nonzero and ultraviolet divergent. But this divergence, and

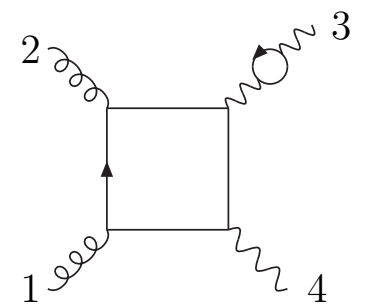

Figure 1: These diagrams give QED divergences. They are exactly canceled by conventional on-shell renormalization. indeed the entire integral, is exactly canceled by the on-shellscheme counterterm, precisely because the external leg is a real, on-shell photon. In the on-shell scheme, the coupling constant should of course be set to $\alpha \equiv \alpha(0)=$ $1 / 137.036 \ldots$ This value should then be used for all the QED couplings associated with real, external photons.

The infrared divergences of a renormalized two-loop amplitude, for the case where the tree amplitude vanishes, are [21],

$$
\left|\mathcal{M}_{n}^{(2)}\left(\mu^{2} ;\{p\}\right)\right\rangle_{\mathrm{RS} .}=\boldsymbol{I}^{(1)}\left(\epsilon, \mu^{2} ;\{p\}\right)\left|\mathcal{M}_{n}^{(1)}\left(\mu^{2} ;\{p\}\right)\right\rangle_{\mathrm{R} . \mathrm{S}}+\left|\mathcal{M}_{n}^{(2) \mathrm{fin}}\left(\mu^{2} ;\{p\}\right)\right\rangle_{\mathrm{R} . \mathrm{S}},
$$


where $\left|\mathcal{M}_{n}^{(L)}\left(\mu^{2} ;\{p\}\right)\right\rangle_{\mathrm{RS} \text {. }}$ is a color space vector representing the renormalized $L$ loop amplitude. The subscript r.s. stands for the choice of renormalization scheme, and $\mu$ is the renormalization scale. These color space vectors give the amplitudes via,

$$
\mathcal{M}_{n}\left(1^{a_{1}}, \ldots, n^{a_{n}}\right) \equiv\left\langle a_{1}, \ldots, a_{n} \mid \mathcal{M}_{n}\left(p_{1}, \ldots, p_{n}\right)\right\rangle
$$

where the $a_{i}$ are color indices. The divergences of $\mathcal{M}_{n}$ are encoded in the color operators $\boldsymbol{I}^{(1)}\left(\epsilon, \mu^{2} ;\{p\}\right)$.

In pure QCD, the operator $\boldsymbol{I}^{(1)}\left(\epsilon, \mu^{2} ;\{p\}\right)$ is given by

$$
\boldsymbol{I}^{(1)}\left(\epsilon, \mu^{2} ;\{p\}\right)=\frac{1}{2} \frac{e^{-\epsilon \psi(1)}}{\Gamma(1-\epsilon)} \sum_{i=1}^{n} \sum_{j \neq i}^{n} \boldsymbol{T}_{i} \cdot \boldsymbol{T}_{j}\left[\frac{1}{\epsilon^{2}}+\frac{\gamma_{i}}{\boldsymbol{T}_{i}^{2}} \frac{1}{\epsilon}\right]\left(\frac{\mu^{2} e^{-i \lambda_{i j} \pi}}{2 p_{i} \cdot p_{j}}\right)^{\epsilon},
$$

where $\lambda_{i j}=+1$ if $i$ and $j$ are both incoming or outgoing partons and $\lambda_{i j}=0$ otherwise. The color charge $\boldsymbol{T}_{i}=\left\{T_{i}^{a}\right\}$ is a vector with respect to the generator label $a$, and an $S U(N)$ matrix with respect to the color indices of the outgoing parton $i$. For external gluons $T_{c b}^{a}=i f^{c a b}$, so $\boldsymbol{T}_{i}^{2}=C_{A}=N$, and

$$
\gamma_{g}=\frac{11}{6} C_{A}-\frac{2}{3} T_{R} N_{f}
$$

The two processes we consider here are

$$
\begin{aligned}
& g\left(-p_{1},-\lambda_{1}\right)+g\left(-p_{2},-\lambda_{2}\right) \rightarrow \gamma\left(p_{3}, \lambda_{3}\right)+\gamma\left(p_{4}, \lambda_{4}\right), \\
& g\left(-p_{1},-\lambda_{1}\right)+\gamma\left(-p_{2},-\lambda_{2}\right) \rightarrow g\left(p_{3}, \lambda_{3}\right)+\gamma\left(p_{4}, \lambda_{4}\right),
\end{aligned}
$$

using an "all-outgoing" convention for the momentum $\left(p_{i}\right)$ and helicity $\left(\lambda_{i}\right)$ labeling. The Mandelstam variables are $s=\left(p_{1}+p_{2}\right)^{2}, t=\left(p_{1}+p_{4}\right)^{2}$, and $u=\left(p_{1}+p_{3}\right)^{2}$. To apply eq. (2.7) to these processes, one may convert two of the gluon legs to photons by setting $\boldsymbol{T}_{\gamma} \cdot \boldsymbol{T}_{i} \rightarrow 0, \boldsymbol{T}_{i} \cdot \boldsymbol{T}_{\gamma} \rightarrow 0$ to obtain the simplified formula

$$
\boldsymbol{I}_{g g \rightarrow \gamma \gamma}^{(1)}\left(\epsilon, \mu^{2} ;\{p\}\right)=-N \frac{e^{-\epsilon \psi(1)}}{\Gamma(1-\epsilon)}\left[\frac{1}{\epsilon^{2}}+\left(\frac{11}{6}-\frac{1}{3} \frac{N_{f}}{N}\right) \frac{1}{\epsilon}\right]\left(\frac{\mu^{2}}{-s}\right)^{\epsilon} .
$$

The color structure of the two-gluon, two-photon amplitude can only be proportional to $\delta^{a_{1} a_{2}}$. Hence $\boldsymbol{I}_{g g \rightarrow \gamma \gamma}^{(1)}$ is necessarily proportional to the identity operator, and the color space language is actually unnecessary. In the $s$-channel where $s>0$ one should use the usual analytic continuation

$$
(-s-i \varepsilon)^{-\epsilon}=|s|^{-\epsilon} e^{i \pi \epsilon \Theta(s)} .
$$

For the crossed process $g \gamma \rightarrow g \gamma$, the same formulae apply with the obvious modification,

$$
\boldsymbol{I}_{g \gamma \rightarrow g \gamma}^{(1)}\left(\epsilon, \mu^{2} ;\{p\}\right)=-N \frac{e^{-\epsilon \psi(1)}}{\Gamma(1-\epsilon)}\left[\frac{1}{\epsilon^{2}}+\left(\frac{11}{6}-\frac{1}{3} \frac{N_{f}}{N}\right) \frac{1}{\epsilon}\right]\left(\frac{\mu^{2}}{-u}\right)^{\epsilon}
$$


The renormalized two-loop amplitudes may thus be separated into divergent parts (which also absorb some finite terms) and a finite remainder,

$$
\begin{aligned}
& \mathcal{M}_{g g \rightarrow \gamma \gamma}^{(2)}=\boldsymbol{I}_{g g \rightarrow \gamma \gamma}^{(1)}\left(\epsilon, \mu^{2} ;\{p\}\right) \mathcal{M}_{g g \rightarrow \gamma \gamma}^{(1)}+\mathcal{M}_{g g \rightarrow \gamma \gamma}^{(2) \mathrm{fin}}, \\
& \mathcal{M}_{g \gamma \rightarrow g \gamma}^{(2)}=\boldsymbol{I}_{g \gamma \rightarrow g \gamma}^{(1)}\left(\epsilon, \mu^{2} ;\{p\}\right) \mathcal{M}_{g \gamma \rightarrow g \gamma}^{(1)}+\mathcal{M}_{g \gamma \rightarrow g \gamma}^{(2) f i n} .
\end{aligned}
$$

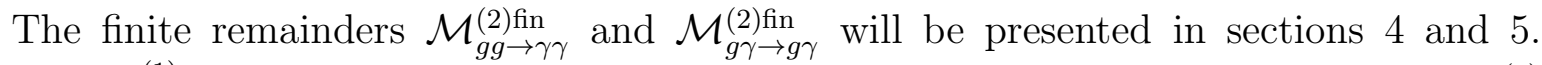
Since $\boldsymbol{I}^{(1)}$ contains $1 / \epsilon^{2}$ poles, the above decomposition requires the expansion of $\mathcal{M}^{(1)}$ through $\mathcal{O}\left(\epsilon^{2}\right)$, which will be given in section 3 .

We present the two-loop amplitudes for definite external helicities, using 't HooftVeltman (HV) dimensional regularization [27]. At one loop, scheme conversions between the various flavors of dimensional regularization have been extensively discussed [28]. Following a similar strategy, one may convert our results in the 't Hooft-Veltman scheme to the conventional dimensional regularization (CDR) scheme. In the CDR scheme one would compute the interference of say $\mathcal{M}_{g g \rightarrow \gamma \gamma}^{(2)}$ with $\mathcal{M}_{g g \rightarrow \gamma \gamma}^{(1)}$, summed over all colors and helicities. This CDR interference may be obtained from our HV result by using eq. (2.14) for $\mathcal{M}_{g g \rightarrow \gamma \gamma}^{(2)}$ and replacing the $\mathrm{HV}$ interference $\mathcal{M}_{g g \rightarrow \gamma \gamma}^{(1)} \times \mathcal{M}_{g g \rightarrow \gamma \gamma}^{(1) *}$ by the same quantity evaluated in the CDR scheme, while leaving $\boldsymbol{I}^{(1)}$ and the finite remain$\operatorname{der} \mathcal{M}_{g g \rightarrow \gamma \gamma}^{(2) \text { fin }}$ of section $⿴$ unaltered. The same considerations apply for $g \gamma \rightarrow g \gamma$ as well. When constructing the cross section for $p p \rightarrow \gamma \gamma X$, the difference between the $\mathrm{HV}$ and the CDR scheme amplitudes is cancelled by a similar scheme dependence in the real emission terms, and at the end only the finite remainder contributes from the two-loop amplitudes [8].

\section{One-loop amplitudes}

As noted above, the decompositions (2.14) and (2.15) require the series expansions of the one-loop $g g \rightarrow \gamma \gamma$ and $g \gamma \rightarrow g \gamma$ amplitudes through $\mathcal{O}\left(\epsilon^{2}\right)$, A simple way to obtain these expansions is to express them in terms of four-gluon amplitudes which are known to all orders in $\epsilon$ [29], in terms of integral functions whose series expansions are known to the appropriate order [10, 11]. The fermion loop contributions to $g g \rightarrow g g$ satisfy the color decomposition

$$
\begin{aligned}
\mathcal{M}_{g g \rightarrow g g}^{(1) f}(1,2,3,4)=N_{f} \sum_{\sigma \in S_{3}} \operatorname{tr}\left[T^{a_{\sigma(1)}} T^{a_{\sigma(2)}} T^{a_{\sigma(3)}} T^{a_{\sigma(4)}}\right] \\
\times A^{(1)}(\sigma(1), \sigma(2), \sigma(3), \sigma(4)),
\end{aligned}
$$

where $N_{f}$ is the number of quark flavors and the sum of permutations $\sigma$ runs over the six non-cyclic permutations of external legs. We have adjusted the normalization factors in the amplitudes to match the conventions of ref. [21. 
One may obtain amplitudes with photons from eq. (3.1) simply by replacing the appropriate color matrices with the identity matrix and altering the normalizations. In particular, the amplitudes where any two legs are gluons and the other two legs are photons are all the same,

$$
\mathcal{M}_{g g \rightarrow \gamma \gamma}^{(1)}=\mathcal{M}_{g \gamma \rightarrow g \gamma}^{(1)}=2 \delta^{a_{1} a_{2}}\left(\sum_{i=1}^{N_{f}} Q_{i}^{2}\right) M^{(1)},
$$

where $Q_{i}$ are the electric charges (2/3 for up-type quarks and $-1 / 3$ for down-type quarks) and

$$
M^{(1)}=\sum_{\sigma \in S_{3}} A^{(1)}(\sigma(1), \sigma(2), \sigma(3), \sigma(4)) .
$$

The permutations $\sigma$ run over the same six orderings of external legs as in eq. (3.1), while the overall factor of 2 arises from our non-standard normalization of color matrices, requiring an extra factor of $\sqrt{2}$ for each gluon converted to a photon. In the permutation sum the ultraviolet and infrared divergences appearing in the four-gluon amplitudes cancel, leaving a finite expression.

The amplitudes are evaluated in the spinor helicity formalism [30]. It is convenient to extract overall spinor phases from each helicity amplitude,

$$
\begin{aligned}
A^{(1)}\left(1^{\lambda_{1}}, 2^{\lambda_{2}}, 3^{\lambda_{3}}, 4^{\lambda_{4}}\right) & =S_{\lambda_{1} \lambda_{2} \lambda_{3} \lambda_{4}} A_{\lambda_{1} \lambda_{2} \lambda_{3} \lambda_{4}}^{(1)}, \\
M^{(1)}\left(1^{\lambda_{1}}, 2^{\lambda_{2}}, 3^{\lambda_{3}}, 4^{\lambda_{4}}\right) & =S_{\lambda_{1} \lambda_{2} \lambda_{3} \lambda_{4}} M_{\lambda_{1} \lambda_{2} \lambda_{3} \lambda_{4}}^{(1)},
\end{aligned}
$$

where the $\lambda_{i}$ signify the helicities of each leg and

$$
\begin{aligned}
S_{++++} & =i \frac{[12][34]}{\langle 12\rangle\langle 34\rangle}, \\
S_{-+++}=i \frac{\langle 12\rangle\langle 14\rangle[24]}{\langle 34\rangle\langle 23\rangle\langle 24\rangle}, & S_{+++}=i \frac{\langle 23\rangle\langle 24\rangle[34]}{\langle 14\rangle\langle 31\rangle\langle 34\rangle}, \\
S_{-+++}=i \frac{\langle 12\rangle[34]}{[12]\langle 34\rangle}, & S_{++-+}=i \frac{\langle 32\rangle\langle 34\rangle[24]}{\langle 14\rangle\langle 21\rangle\langle 24\rangle}, \\
S_{-++} & =i \frac{\langle 13\rangle[24]}{[13]\langle 24\rangle},
\end{aligned}
$$

Our conventions here are that all external legs are outgoing; for incoming legs one should reverse the helicities of those legs. The spinor inner products [30, 31] are $\langle i j\rangle=\left\langle i^{-} \mid j^{+}\right\rangle$ and $[i j]=\left\langle i^{+} \mid j^{-}\right\rangle$, where $\left|i^{ \pm}\right\rangle$are massless Weyl spinors of momentum $k_{i}$, labeled with the sign of the helicity. They are anti-symmetric, with norm $|\langle i j\rangle|=|[i j]|=\sqrt{s_{i j}}$, where $s_{i j}=2 k_{i} \cdot k_{j}$. From these definitions it follows that the $S_{\lambda_{1} \lambda_{2} \lambda_{3} \lambda_{4}}$ are phases.

The fermion loop contributions to the one-loop four-gluon helicity amplitudes, in a form valid to all orders in $\epsilon$ (with four-dimensional external momenta $p_{i}$ ) are [29]

$$
A_{++++}^{(1)}=\epsilon(1-\epsilon) \operatorname{Box}^{(8)}(s, t),
$$




$$
\begin{aligned}
A_{-+++}^{(1)}= & -\frac{t(u-s)}{s u} \epsilon \operatorname{Tri}^{(6)}(s)-\frac{s(u-t)}{t u} \epsilon \operatorname{Tri}^{(6)}(t) \\
& -\frac{t-u}{s^{2}} \epsilon \operatorname{Bub}^{(6)}(s)-\frac{s-u}{t^{2}} \epsilon \operatorname{Bub}^{(6)}(t)+\frac{s t}{2 u} \epsilon \operatorname{Box}^{(6)}(s, t) \\
& +\epsilon(1-\epsilon) \operatorname{Box}^{(8)}(s, t) \\
A_{++-+}^{(1)}= & A_{-+++}^{(1)} \\
A_{--++}^{(1)}=- & \frac{1}{2} s \epsilon \operatorname{Box}^{(6)}(s, t)-\frac{s}{2 t} \operatorname{Bub}^{(4)}(t) \\
& +\frac{s}{t^{2}} \operatorname{Bub}^{(6)}(t)-\frac{1}{t} \epsilon \operatorname{Bub}^{(6)}(t)+\epsilon(1-\epsilon) \operatorname{Box}^{(8)}(s, t), \\
A_{-+-+}^{(1)}= & \frac{u}{s t}\left[\frac{1}{2} t \operatorname{Bub}^{(4)}(s)+\frac{1}{2} s \operatorname{Bub}^{(4)}(t)-\frac{1}{2} s t(1-\epsilon) \operatorname{Box}^{(6)}(s, t)\right. \\
& +\frac{s t(s-t)}{u^{2}} \epsilon \operatorname{Tri}^{(6)}(t)+\frac{s t(t-s)}{u^{2}} \epsilon \operatorname{Tri}^{(6)}(s)-\frac{t s^{2}}{u^{2}} \operatorname{Bub}^{(4)}(t) \\
& -\frac{s t^{2}}{u^{2}} \operatorname{Bub}^{(4)}(s)-\frac{s}{t} \operatorname{Bub}^{(6)}(t)-\frac{t}{s} \operatorname{Bub}^{(6)}(s)+\frac{s}{u} \epsilon \operatorname{Bub}^{(6)}(t) \\
& +\frac{t}{u} \epsilon \operatorname{Bub}^{(6)}(s)+\frac{s t}{u} \operatorname{Tri}^{(6)}(t)+\frac{s t}{u} \operatorname{Tri}^{(6)}(s) \\
& \left.+\frac{s^{2} t^{2}}{u^{2}} \operatorname{Box}^{(6)}(s, t)+\frac{s t}{u} \epsilon(1-\epsilon) \operatorname{Box}^{(8)}(s, t)\right] .
\end{aligned}
$$

Here $\operatorname{Bub}^{(n)}(s)$, Tri ${ }^{(n)}(s)$ and $\operatorname{Box}^{(n)}(s, t)$ are the one-loop bubble, triangle and box scalar integrals, evaluated in $D=n-2 \epsilon$ dimensions. The remaining helicity configurations can all be obtained using parity and relabelings. In the CDR scheme one would need additional $\epsilon$-helicities 32.

The bubble and box integrals that appear in the above amplitudes are

$$
\begin{aligned}
\operatorname{Bub}^{(4)}(s) & =\frac{r_{\Gamma}}{\epsilon(1-2 \epsilon)}(-s)^{-\epsilon}, \\
\operatorname{Bub}^{(6)}(s) & =-\frac{r_{\Gamma}}{2 \epsilon(1-2 \epsilon)(3-2 \epsilon)}(-s)^{1-\epsilon}, \\
\operatorname{Tri}^{(4)}(s) & =-\frac{r_{\Gamma}}{\epsilon^{2}}(-s)^{-1-\epsilon}, \\
\operatorname{Tri}^{(6)}(s) & =-\frac{r_{\Gamma}(-s)^{-\epsilon}}{2 \epsilon(1-2 \epsilon)(1-\epsilon)},
\end{aligned}
$$

where

$$
\begin{aligned}
r_{\Gamma} & =e^{-\epsilon \psi(1)} \frac{\Gamma(1+\epsilon) \Gamma^{2}(1-\epsilon)}{\Gamma(1-2 \epsilon)} \\
& =1-\frac{1}{2} \zeta_{2} \epsilon^{2}-\frac{7}{3} \zeta_{3} \epsilon^{3}-\frac{47}{16} \zeta_{4} \epsilon^{4}+\mathcal{O}\left(\epsilon^{5}\right),
\end{aligned}
$$


with

$$
\zeta_{s} \equiv \sum_{n=1}^{\infty} n^{-s}, \quad \zeta_{2}=\frac{\pi^{2}}{6}, \quad \zeta_{3}=1.202057 \ldots, \quad \zeta_{4}=\frac{\pi^{4}}{90}
$$

and we have kept the full dependence on $\epsilon$ in the integrals. In the $s$-channel where $s>0$ the functions are given by the analytic continuation (2.12).

The box integrals in various dimensions appearing in eq. (3.6) are related via a dimension-shifting formula [33] valid to all orders in $\epsilon$,

$$
\begin{aligned}
& \operatorname{Box}^{(6)}(s, t)=\frac{1}{2(-1+2 \epsilon) u}\left(s t \operatorname{Box}^{(4)}(s, t)-2 t \operatorname{Tri}^{(4)}(t)-2 s \operatorname{Tri}^{(4)}(s)\right), \\
& \operatorname{Box}^{(8)}(s, t)=\frac{1}{2(-3+2 \epsilon) u}\left(s t \operatorname{Box}^{(6)}(s, t)-2 t \operatorname{Tri}^{(6)}(t)-2 s \operatorname{Tri}^{(6)}(s)\right) .
\end{aligned}
$$

Since the $D=6-2 \epsilon$ scalar box integral is completely finite as $\epsilon \rightarrow 0$, it is convenient to express the other box integrals in terms of it. This isolates all divergences to triangle and bubble integrals. In the $u$-channel $(s<0, t<0)$, where the functions are manifestly real, the expansion of the six-dimensional box through $\mathcal{O}\left(\epsilon^{2}\right)$ is [10, 11]

$$
\begin{aligned}
\operatorname{Box}^{(6)}(s, t)= & \frac{r_{\Gamma} u^{-1-\epsilon}}{2(1-2 \epsilon)}\left[\frac{1}{2}\left((V-W)^{2}+\pi^{2}\right)\right. \\
& +2 \epsilon\left(\operatorname{Li}_{3}(-v)-V \operatorname{Li}_{2}(-v)-\frac{V^{3}}{3}-\frac{\pi^{2}}{2} V\right) \\
& -2 \epsilon^{2}\left(\operatorname{Li}_{4}(-v)+W \operatorname{Li}_{3}(-v)-\frac{1}{2} V^{2} \operatorname{Li}_{2}(-v)-\frac{1}{8} V^{4}-\frac{1}{6} V^{3} W\right. \\
& \left.\left.+\frac{1}{4} V^{2} W^{2}-\frac{\pi^{2}}{4} V^{2}-\frac{\pi^{2}}{3} V W-2 \zeta_{4}\right)+(s \leftrightarrow t)\right]+\mathcal{O}\left(\epsilon^{3}\right),
\end{aligned}
$$

where

$$
v=\frac{s}{u}, \quad w=\frac{t}{u}, \quad V=\ln \left(-\frac{s}{u}\right), \quad W=\ln \left(-\frac{t}{u}\right) .
$$

In the $s$-channel $(s>0, t<0)$ an analytic continuation of the box integral yields,

$$
\begin{aligned}
\operatorname{Box}^{(6)}(s, t)= & \frac{r_{\Gamma}|s|^{-\epsilon}}{u(1-2 \epsilon)}\left\{\frac{1}{2} X^{2}\right. \\
& +\epsilon\left(-\operatorname{Li}_{3}(-x)+X \operatorname{Li}_{2}(-x)-\frac{1}{3} X^{3}+\zeta_{3}+\frac{1}{2} Y X^{2}-\frac{1}{2} \pi^{2} X\right) \\
& -\epsilon^{2}\left(\operatorname{Li}_{4}(-x / y)-\operatorname{Li}_{4}(-y)+\operatorname{Li}_{3}(-y) X\right. \\
& +\frac{1}{2} \operatorname{Li}_{2}(-x)\left(X^{2}+\pi^{2}\right)+\frac{1}{24}\left(Y^{2}+\pi^{2}\right)^{2}-\frac{1}{6} Y^{3} X+\frac{1}{4} Y^{2} X^{2}
\end{aligned}
$$




$$
\begin{aligned}
& \left.+\frac{1}{3} X^{3} Y-\frac{1}{8}\left(X^{2}+\pi^{2}\right)^{2}+\frac{\pi^{2}}{3} X Y+\frac{7}{360} \pi^{4}\right) \\
& +i \pi\left[X+\epsilon\left(\operatorname{Li}_{2}(-x)+Y X-\frac{1}{2} X^{2}-\frac{\pi^{2}}{6}\right)\right. \\
& \left.\left.+\epsilon^{2}\left(-\operatorname{Li}_{3}(-x)-\operatorname{Li}_{3}(-y)-\frac{1}{2} Y X^{2}+\frac{1}{6} X^{3}+\zeta_{3}\right)\right]\right\} \\
& +\mathcal{O}\left(\epsilon^{3}\right),
\end{aligned}
$$

where the variables appearing in the amplitudes are

$$
x=\frac{t}{s}, \quad y=\frac{u}{s}, \quad X=\ln \left(-\frac{t}{s}\right), \quad Y=\ln \left(-\frac{u}{s}\right) .
$$

In the permutation sum appearing in eq. (3.2), after appropriate relabelings, both expansions of the box integrals (3.12) and (3.14) appear.

Through $\mathcal{O}\left(\epsilon^{0}\right)$ the amplitudes simply greatly and the $M_{\lambda_{1} \lambda_{2} \lambda_{3} \lambda_{4}}^{(1)}$ reduce to,

$$
\begin{aligned}
M_{++++}^{(1)} & =1+\mathcal{O}(\epsilon), \\
M_{-+++}^{(1)} & =M_{+-++}^{(1)}=M_{++-+}^{(1)}=M_{+++-}^{(1)}=1+\mathcal{O}(\epsilon), \\
M_{--++}^{(1)} & =-\frac{1}{2} \frac{t^{2}+u^{2}}{s^{2}}\left[\ln ^{2}\left(\frac{t}{u}\right)+\pi^{2}\right]-\frac{t-u}{s} \ln \left(\frac{t}{u}\right)-1+\mathcal{O}(\epsilon), \\
M_{-+-+}^{(1)} & =-\frac{1}{2} \frac{t^{2}+s^{2}}{u^{2}} \ln ^{2}\left(-\frac{t}{s}\right)-\frac{t-s}{u} \ln \left(-\frac{t}{s}\right)-1 \\
-i \pi\left[\frac{t^{2}+s^{2}}{u^{2}} \ln \left(-\frac{t}{s}\right)+\frac{t-s}{u}\right]+\mathcal{O}(\epsilon), & \\
M_{+--+}^{(1)}(s, t, u) & =M_{-+-+}^{(1)}(s, u, t) .
\end{aligned}
$$

\section{Finite parts of the two-loop $g g \rightarrow \gamma \gamma$ amplitudes}

A generic sample of two-loop Feynman diagrams for $g g \rightarrow \gamma \gamma$ is shown in Figure 2. We did not evaluate the diagrams directly. Instead we computed the unitarity cuts in various channels, working to all orders in the dimensional regularization parameter $\epsilon=(4-D) / 2$ [14]. Essentially we followed the approach first employed at two loops for the pure gluon four-point amplitude with all identical helicities [10 and for $N=4$ supersymmetric amplitudes [9]. These amplitudes were simple enough that a compact expression for the integrand could be given. The fermion loop contributions with all plus helicities are about as simple [34]. However, for the generic helicity configuration, the integrands become rather complicated. We therefore used the general integral reduction algorithms developed for the all-massless four-point topologies [17, 18, 19], in order to reduce the loop integrals to a minimal basis of master integrals. To incorporate 
polarization vectors of photons and gluons with definite helicity requires some minor extensions of these techniques [35].
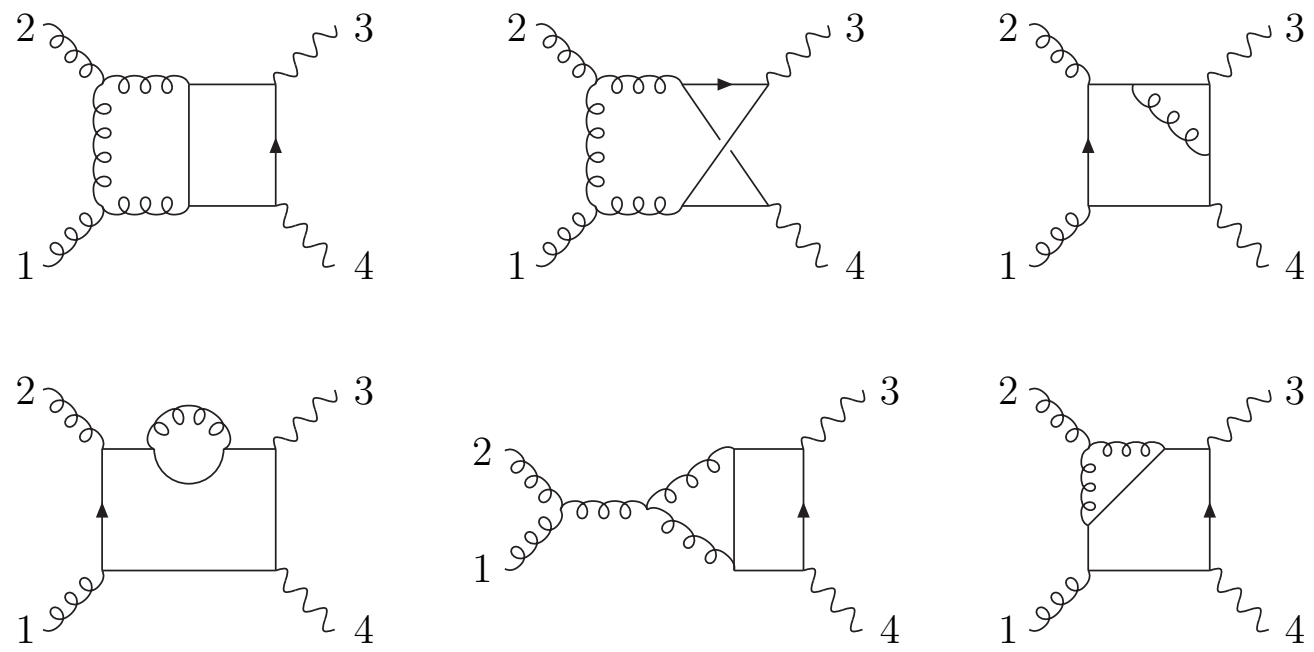

Figure 2: Some of the two-loop diagrams for $g g \rightarrow \gamma \gamma$. The curly lines represent gluons while the wavy ones photons.

We then expand the master integrals in a Laurent series in $\epsilon$, which begins at order $1 / \epsilon^{4}$. Many of the master integral expansions quoted in refs. [15, 16, 17, 18, 20] are in terms of Nielsen functions [36],

$$
S_{n, p}(x)=\frac{(-1)^{n+p-1}}{(n-1) ! p !} \int_{0}^{1} \frac{d t}{t} \ln ^{n-1} t \ln ^{p}(1-x t),
$$

with $n+p \leq 4$. We have found it useful to express the results instead in terms of a minimal set of polylogarithms [37,

$$
\begin{aligned}
& \operatorname{Li}_{n}(x)=\sum_{i=1}^{\infty} \frac{x^{i}}{i^{n}}=\int_{0}^{x} \frac{d t}{t} \operatorname{Li}_{n-1}(t), \\
& \operatorname{Li}_{2}(x)=-\int_{0}^{x} \frac{d t}{t} \ln (1-t)
\end{aligned}
$$

with $n=2,3,4$, using relations such as 38

$$
\begin{aligned}
S_{13}(x)=-\mathrm{Li}_{4}(1-x)+\ln (1-x) \operatorname{Li}_{3}(1-x)+\frac{1}{2} \ln ^{2}(1-x)\left(\operatorname{Li}_{2}(x)-\zeta_{2}\right) & \\
+ & \frac{1}{3} \ln ^{3}(1-x) \ln x+\zeta_{4} \\
S_{22}(x)=\mathrm{Li}_{4}(x) & -\mathrm{Li}_{4}(1-x)+\operatorname{Li}_{4}\left(\frac{-x}{1-x}\right)-\ln (1-x)\left(\operatorname{Li}_{3}(x)-\zeta_{3}\right)
\end{aligned}
$$




$$
\begin{aligned}
& +\frac{1}{24} \ln ^{4}(1-x)-\frac{1}{6} \ln ^{3}(1-x) \ln x+\frac{1}{2} \zeta_{2} \ln ^{2}(1-x)+\zeta_{4}, \\
& \text { for } 0<x<1
\end{aligned}
$$

The analytic properties of the non-planar double box integrals appearing in the amplitudes are somewhat intricate [10, 16], since there is no Euclidean region in any of the three kinematic channels for the $2 \rightarrow 2$ process. We quote our results in the physical $s$-channel $(s>0 ; t, u<0)$ for the $g g \rightarrow \gamma \gamma$ kinematics (2.9).

The dependence of the the finite remainder in eq. (2.14) on quark charges, the renormalization scale $\mu, N$ and $N_{f}$ may be extracted as,

$$
\begin{gathered}
\mathcal{M}_{g g \rightarrow \gamma \gamma}^{(2) \mathrm{fin}}=2 \delta^{a b}\left(\sum_{j=1}^{N_{f}} Q_{j}^{2}\right) S_{\lambda_{1} \lambda_{2} \lambda_{3} \lambda_{4}}\left[\frac{11 N-2 N_{f}}{6}\left(\ln \left(\mu^{2} / s\right)+i \pi\right) M_{\lambda_{1} \lambda_{2} \lambda_{3} \lambda_{4}}^{(1)}\right. \\
\left.+N F_{\lambda_{1} \lambda_{2} \lambda_{3} \lambda_{4}}^{\mathrm{L}}-\frac{1}{N} F_{\lambda_{1} \lambda_{2} \lambda_{3} \lambda_{4}}^{\mathrm{SL}}\right],
\end{gathered}
$$

where the spinor phases are defined in eq. (3.5) and coupling constants have been extracted in eq. (2.3). The $\mu$-dependence in the first term in this expression is a consequence of renormalization group invariance.

The two-loop QED corrections to $g g \rightarrow \gamma \gamma$ require the same set of two-loop diagrams as the subleading-color QCD corrections. In the QED case, external fermion bubble insertions on the photon legs should be added. However, as mentioned in section 3 these diagrams are exactly cancelled by the ultraviolet counterterm in the usual on-shell renormalization of QED (just as in the light-by-light case [39]). The renormalized amplitudes are free of infrared and ultraviolet divergences and are given by

$$
\mathcal{M}_{g g \rightarrow \gamma \gamma}^{(2) \mathrm{QED}}=4 \delta^{a b}\left(\sum_{j=1}^{N_{f}} Q_{j}^{4}\right) S_{\lambda_{1} \lambda_{2} \lambda_{3} \lambda_{4}} F_{\lambda_{1} \lambda_{2} \lambda_{3} \lambda_{4}}^{\mathrm{SL}},
$$

where a factor of $\alpha\left(\mu^{2}\right)$ replaces one factor of $\alpha_{s}\left(\mu^{2}\right)$ in the prefactor in front of $\mathcal{M}^{(2)}$ in eq. (2.3). The two-loop QCD and QED corrections to light-by-light scattering via massless fermions are also proportional to $F_{\lambda_{1} \lambda_{2} \lambda_{3} \lambda_{4}}^{\mathrm{SL}}$ [39]. (In ref. [39] particles 1 and 2 are taken to be incoming, so the helicity labels for legs 1 and 2 are reversed with respect to the labeling used here.)

The explicit forms for the $F_{\lambda_{1} \lambda_{2} \lambda_{3} \lambda_{4}}^{\mathrm{L}}$ appearing in eq. (4.5) are

$$
\begin{aligned}
& F_{++++}^{\mathrm{L}}= \frac{1}{2}, \\
& F_{-+++}^{\mathrm{L}}=\frac{1}{8}\left[\left(2+4 \frac{x}{y^{2}}-5 \frac{x^{2}}{y^{2}}\right)\left((X+i \pi)^{2}+\pi^{2}\right)-(1-x y)\left((X-Y)^{2}+\pi^{2}\right)\right. \\
&\left.+2\left(\frac{9}{y}-10 x\right)(X+i \pi)\right]+\{t \leftrightarrow u\},
\end{aligned}
$$




$$
\begin{aligned}
& F_{++-+}^{\mathrm{L}}=\frac{1}{8}\left[\left(2+6 \frac{x}{y^{2}}-3 \frac{x^{2}}{y^{2}}\right)\left((X+i \pi)^{2}+\pi^{2}\right)-(x-y)^{2}\left((X-Y)^{2}+\pi^{2}\right)\right. \\
& \left.+2\left(\frac{9}{y}-8 x\right)(X+i \pi)\right]+\{t \leftrightarrow u\} \\
& F_{--++}^{\mathrm{L}}=-\left(x^{2}+y^{2}\right)\left[4 \operatorname{Li}_{4}(-x)+(Y-3 X-2 i \pi) \operatorname{Li}_{3}(-x)\right. \\
& +\left((X+i \pi)^{2}+\pi^{2}\right) \operatorname{Li}_{2}(-x)+\frac{1}{48}(X+Y)^{4} \\
& \left.+i \frac{\pi}{12}(X+Y)^{3}+i \frac{\pi^{3}}{2} X-\frac{\pi^{2}}{12} X^{2}-\frac{109}{720} \pi^{4}\right] \\
& +\frac{1}{2} x(1-3 y)\left[\operatorname{Li}_{3}(-x / y)-(X-Y) \operatorname{Li}_{2}(-x / y)-\zeta_{3}+\frac{1}{2} Y\left((X-Y)^{2}+\pi^{2}\right)\right] \\
& +\frac{1}{4} x^{2}\left[(X-Y)^{3}+3(Y+i \pi)\left((X-Y)^{2}+\pi^{2}\right)\right] \\
& +\frac{1}{8}\left(14(x-y)-\frac{8}{y}+\frac{9}{y^{2}}\right)\left((X+i \pi)^{2}+\pi^{2}\right) \\
& +\frac{1}{16}(38 x y-13)\left((X-Y)^{2}+\pi^{2}\right)-\frac{\pi^{2}}{12}-\frac{9}{4}\left(\frac{1}{y}+2 x\right)(X+i \pi)+\frac{1}{4} \\
& +\{t \leftrightarrow u\} \\
& F_{-+-+}^{\mathrm{L}}=-2 \frac{x^{2}+1}{y^{2}}\left[\operatorname{Li}_{4}(-x)-\zeta_{4}-\frac{1}{2}(X+i \pi)\left(\operatorname{Li}_{3}(-x)-\zeta_{3}\right)\right. \\
& +\frac{\pi^{2}}{6}\left(\operatorname{Li}_{2}(-x)-\frac{\pi^{2}}{6}-\frac{1}{2} X^{2}\right)-\frac{1}{48} X^{4} \\
& \left.+\frac{1}{24}(X+i \pi)^{2}\left((X+i \pi)^{2}+\pi^{2}\right)\right] \\
& +2 \frac{3(1-x)^{2}-2}{y^{2}}\left[\operatorname{Li}_{4}(-x)+\operatorname{Li}_{4}(-x / y)-\operatorname{Li}_{4}(-y)-(Y+i \pi)\left(\operatorname{Li}_{3}(-x)-\zeta_{3}\right)\right. \\
& \left.+\frac{\pi^{2}}{6}\left(\operatorname{Li}_{2}(-x)+\frac{1}{2} Y^{2}\right)-\frac{1}{6} X Y^{3}+\frac{1}{24} Y^{4}-\frac{7}{360} \pi^{4}\right] \\
& -\frac{2}{3}\left(8-x+30 \frac{x}{y}\right)\left[\operatorname{Li}_{3}(-y)-\zeta_{3}-(Y+i \pi)\left(\operatorname{Li}_{2}(-y)-\frac{\pi^{2}}{6}\right)\right. \\
& \left.-\frac{1}{2} X\left((Y+i \pi)^{2}+\pi^{2}\right)\right] \\
& +\frac{1}{6}\left(4 y+27+\frac{42}{y}+\frac{4}{y^{2}}\right)\left[\operatorname{Li}_{3}(-x)-\zeta_{3}-(X+i \pi)\left(\operatorname{Li}_{2}(-x)-\frac{\pi^{2}}{6}\right)\right. \\
& \left.+i \frac{\pi}{2} X^{2}-\pi^{2} X\right]
\end{aligned}
$$




$$
\begin{aligned}
& +\frac{1}{12}\left(3-\frac{2}{y}-12 \frac{x}{y^{2}}\right)(X+i \pi)\left((X+i \pi)^{2}+\pi^{2}\right) \\
& -\frac{1}{3} y(X+i \pi)\left((Y+i \pi)^{2}+\pi^{2}\right)+2\left(1+\frac{2}{y}\right)\left(\zeta_{3}-\frac{\pi^{2}}{6}(Y+i \pi)\right) \\
& +\frac{1}{24}\left(y^{2}-24 y+44-8 \frac{x^{3}}{y}\right)\left((X-Y)^{2}+\pi^{2}\right) \\
& -\frac{1}{24}\left(15-14 \frac{x}{y}-48 \frac{x}{y^{2}}\right)\left((X+i \pi)^{2}+\pi^{2}\right) \\
& +\frac{1}{24}\left(8 \frac{x}{y}+60-24 \frac{y}{x}+27 \frac{y^{2}}{x^{2}}\right)\left((Y+i \pi)^{2}+\pi^{2}\right)+\frac{4}{9} \pi^{2} \frac{x}{y} \\
& +\frac{1}{12}\left(2 x^{2}-54 x-27 y^{2}\right)\left(\frac{1}{y}(X+i \pi)+\frac{1}{x}(Y+i \pi)\right),
\end{aligned}
$$

where the last amplitude does not possess symmetry under $t \leftrightarrow u$. In contrast to the one-loop case, the -+++ case is distinct from the ++-+ case, because at two loops external gluons can couple to a gluon internal to the diagram via the non-abelian coupling, while photons cannot.

Similarly, the subleading color contributions in eq. (4.5) are expressed in terms of the functions,

$$
\begin{aligned}
& F_{++++}^{\mathrm{SL}}=-\frac{3}{2} \\
& F_{-+++}^{\mathrm{SL}}=\frac{1}{8}\left[\frac{x^{2}+1}{y^{2}}\left((X+i \pi)^{2}+\pi^{2}\right)+\frac{1}{2}\left(x^{2}+y^{2}\right)\left((X-Y)^{2}+\pi^{2}\right)\right. \\
& \left.-4\left(\frac{1}{y}-x\right)(X+i \pi)\right]+\{t \leftrightarrow u\} \\
& F_{++-+}^{\mathrm{SL}}=F_{+-++}^{\mathrm{SL}}=F_{+++-}^{\mathrm{SL}}=F_{-+++}^{\mathrm{SL}}, \\
& F_{--++}^{\mathrm{SL}}=-2 x^{2}\left[\operatorname{Li}_{4}(-x)+\mathrm{Li}_{4}(-y)-(X+i \pi)\left(\operatorname{Li}_{3}(-x)+\operatorname{Li}_{3}(-y)\right)\right. \\
& \left.+\frac{1}{12} X^{4}-\frac{1}{3} X^{3} Y+\frac{\pi^{2}}{12} X Y-\frac{4}{90} \pi^{4}+i \frac{\pi}{6} X\left(X^{2}-3 X Y+\pi^{2}\right)\right] \\
& -(x-y)\left(\operatorname{Li}_{4}(-x / y)-\frac{\pi^{2}}{6} \operatorname{Li}_{2}(-x)\right) \\
& -x\left[2 \mathrm{Li}_{3}(-x)-\mathrm{Li}_{3}(-x / y)-3 \zeta_{3}-2(X+i \pi) \mathrm{Li}_{2}(-x)\right. \\
& +(X-Y)\left(\operatorname{Li}_{2}(-x / y)+X^{2}\right)+\frac{1}{12}(5(X-Y)+18 i \pi)\left((X-Y)^{2}+\pi^{2}\right)
\end{aligned}
$$




$$
\begin{aligned}
& \left.-\frac{2}{3} X\left(X^{2}+\pi^{2}\right)-i \pi\left(Y^{2}+\pi^{2}\right)\right] \\
& +\frac{1-2 x^{2}}{4 y^{2}}\left((X+i \pi)^{2}+\pi^{2}\right)-\frac{1}{8}(2 x y+3)\left((X-Y)^{2}+\pi^{2}\right)+\frac{\pi^{2}}{12} \\
& +\left(\frac{1}{2 y}+x\right)(X+i \pi)-\frac{1}{4}+\{t \leftrightarrow u\} \\
& F_{-+-+}^{\mathrm{SL}}=-2 \frac{x^{2}+1}{y^{2}}\left[\operatorname{Li}_{4}(-x / y)-\operatorname{Li}_{4}(-y)+\frac{1}{2}(X-2 Y-i \pi)\left(\operatorname{Li}_{3}(-x)-\zeta_{3}\right)\right. \\
& \left.+\frac{1}{24}\left(X^{4}+2 i \pi X^{3}-4 X Y^{3}+Y^{4}+2 \pi^{2} Y^{2}\right)+\frac{7}{360} \pi^{4}\right] \\
& -2 \frac{x-1}{y}\left[\mathrm{Li}_{4}(-x)-\zeta_{4}-\frac{1}{2}(X+i \pi)\left(\operatorname{Li}_{3}(-x)-\zeta_{3}\right)\right. \\
& \left.+\frac{\pi^{2}}{6}\left(\operatorname{Li}_{2}(-x)-\frac{\pi^{2}}{6}-\frac{1}{2} X^{2}\right)-\frac{1}{48} X^{4}\right] \\
& +\left(2 \frac{x}{y}-1\right)\left[\operatorname{Li}_{3}(-x)-(X+i \pi) \operatorname{Li}_{2}(-x)+\zeta_{3}-\frac{1}{6} X^{3}-\frac{\pi^{2}}{3}(X+Y)\right] \\
& +2\left(2 \frac{x}{y}+1\right)\left[\operatorname{Li}_{3}(-y)+(Y+i \pi) \operatorname{Li}_{2}(-x)-\zeta_{3}+\frac{1}{4} X\left(2 Y^{2}+\pi^{2}\right)\right. \\
& \left.-\frac{1}{8} X^{2}(X+3 i \pi)\right]-\frac{1}{4}\left(2 x^{2}-y^{2}\right)\left((X-Y)^{2}+\pi^{2}\right) \\
& -\frac{1}{4}\left(3+2 \frac{x}{y^{2}}\right)\left((X+i \pi)^{2}+\pi^{2}\right)-\frac{2-y^{2}}{4 x^{2}}\left((Y+i \pi)^{2}+\pi^{2}\right)+\frac{\pi^{2}}{6} \\
& +\frac{1}{2}\left(2 x+y^{2}\right)\left[\frac{1}{y}(X+i \pi)+\frac{1}{x}(Y+i \pi)\right]-\frac{1}{2}, \\
& F_{+--+}^{\mathrm{SL}}(s, t, u)=F_{-+-+}^{\mathrm{SL}}(s, u, t) .
\end{aligned}
$$

The variables appearing in the amplitudes are defined in eq. (3.15).

\section{Finite parts of the two-loop $g \gamma \rightarrow g \gamma$ amplitudes}

The finite remainder for the $g \gamma \rightarrow g \gamma$ amplitude is defined in eq. (2.15). Its dependence on quark charges, the renormalization scale $\mu, N$ and $N_{f}$ is given by

$$
\begin{array}{r}
\mathcal{M}_{g \gamma \rightarrow g \gamma}^{(2) \operatorname{fin}}=2 \delta^{a b}\left(\sum_{j=1}^{N_{f}} Q_{j}^{2}\right) S_{\lambda_{1} \lambda_{2} \lambda_{3} \lambda_{4}}\left[\frac{11 N-2 N_{f}}{6} \ln \left(-\mu^{2} / u\right) M_{\lambda_{1} \lambda_{2} \lambda_{3} \lambda_{4}}^{(1)}\right. \\
\left.+N G_{\lambda_{1} \lambda_{2} \lambda_{3} \lambda_{4}}^{\mathrm{L}}-\frac{1}{N} F_{\lambda_{1} \lambda_{2} \lambda_{3} \lambda_{4}}^{\mathrm{SL}}\right] .
\end{array}
$$


In this case legs 1,3 are the gluons and legs 2,4 are the photons. The subleading-color functions $F_{\lambda_{1} \lambda_{2} \lambda_{3} \lambda_{4}}^{\mathrm{SL}}$ are the same as for $g g \rightarrow \gamma \gamma$.

The leading-color functions $G_{\lambda_{1} \lambda_{2} \lambda_{3} \lambda_{4}}^{\mathrm{L}}$ are new, and are given by

$$
\begin{aligned}
G_{++++}^{\mathrm{L}}= & \frac{1}{2} \\
G_{-+++}^{\mathrm{L}}= & \frac{1}{8}\left[\left(2+4 x y-5 x^{2}\right)\left((X-Y)^{2}+\pi^{2}\right)+\left(2+4 \frac{y}{x^{2}}-\frac{5}{x^{2}}\right)\left((Y+i \pi)^{2}+\pi^{2}\right)\right. \\
& \left.-2\left(1-\frac{x}{y^{2}}\right)\left((X+i \pi)^{2}+\pi^{2}\right)+2\left(9-10 \frac{x}{y^{2}}\right)\left(y(X-Y)-\frac{y}{x}(Y+i \pi)\right)\right], \\
G_{+-++}^{\mathrm{L}}= & \frac{1}{8}\left[\left(2+6 x y-3 x^{2}\right)\left((X-Y)^{2}+\pi^{2}\right)+\left(2+6 \frac{y}{x^{2}}-\frac{3}{x^{2}}\right)\left((Y+i \pi)^{2}+\pi^{2}\right)\right. \\
& \left.-2 \frac{(1-x)^{2}}{y^{2}}\left((X+i \pi)^{2}+\pi^{2}\right)+2\left(9-8 \frac{x}{y^{2}}\right)\left(y(X-Y)-\frac{y}{x}(Y+i \pi)\right)\right]
\end{aligned}
$$

$$
\begin{aligned}
G_{--++}^{\mathrm{L}}= & -2\left(x^{2}+y^{2}\right)\left[\operatorname{Li}_{4}(-x / y)-\frac{1}{2}(X-Y)\left(\operatorname{Li}_{3}(-x / y)-\zeta_{3}\right)+\frac{\pi^{2}}{6}\left(\operatorname{Li}_{2}(-x / y)\right.\right. \\
& \left.\left.+\frac{1}{2}(X-Y)^{2}\right)+\frac{1}{48}(X-Y)^{4}-i \frac{\pi}{12}(X-Y)\left((X-Y)^{2}+\pi^{2}\right)+\frac{17}{720} \pi^{4}\right] \\
+ & 2\left(3(x-y)^{2}-2 y^{2}\right)\left[\operatorname{Li}_{4}(-x / y)+\operatorname{Li}_{4}(-x)+\mathrm{Li}_{4}(-y)\right. \\
& +(Y+i \pi)\left(\operatorname{Li}_{3}(-x / y)-\zeta_{3}\right)+\frac{\pi^{2}}{6}\left(\operatorname{Li}_{2}(-x / y)-\frac{1}{2} Y^{2}\right) \\
& \left.+\frac{1}{6}(X-Y) Y^{3}+\frac{1}{12} Y^{4}-\frac{\pi^{4}}{24}\right] \\
- & \frac{2}{3}\left(8-\frac{x}{y}+30 x\right)\left[\operatorname{Li}_{3}(-y)-\zeta 3-(Y+i \pi)\left(\operatorname{Li}_{2}(-y)-\frac{\pi^{2}}{6}\right)\right. \\
& \left.-\frac{1}{2}(X-Y)\left((Y+i \pi)^{2}+\pi^{2}\right)-\frac{1}{3} Y\left(Y^{2}+\frac{3}{2} i \pi Y+\pi^{2}\right)\right] \\
+ & \frac{1}{6}\left(\frac{4}{y}+27+42 y+4 y^{2}\right)\left[\operatorname{Li}_{3}(-x / y)-\zeta_{3}-(X-Y)\left(\operatorname{Li}_{2}(-x / y)-\frac{\pi^{2}}{6}\right)\right. \\
& \left.-i \frac{\pi}{2}\left((X-Y)^{2}+\pi^{2}\right)\right] \\
+ & \frac{1}{12}(3-2 y-12 x y)(X-Y)\left((X-Y)^{2}+\pi^{2}\right)
\end{aligned}
$$




$$
\begin{aligned}
& -\frac{1}{3 y}(X-Y)\left((Y+i \pi)^{2}+\pi^{2}\right)+2(1+2 y)\left(\zeta_{3}+\frac{\pi^{2}}{6}(Y+i \pi)\right) \\
& +\frac{1}{24}\left(\frac{1}{y^{2}}-\frac{24}{y}+44-8 \frac{x^{3}}{y^{2}}\right)\left((X+i \pi)^{2}+\pi^{2}\right) \\
& -\frac{1}{24}(15-14 x-48 x y)\left((X-Y)^{2}+\pi^{2}\right) \\
& +\frac{1}{24}\left(8 x+60-\frac{24}{x}+\frac{27}{x^{2}}\right)\left((Y+i \pi)^{2}+\pi^{2}\right)+\frac{4}{9} \pi^{2} x \\
& +\frac{1}{12} \frac{2 x^{2}-54 x y-27}{y}\left(X-Y-\frac{1}{x}(Y+i \pi)\right) \text {, } \\
& G_{-+-+}^{\mathrm{L}}=\left(1-2 \frac{x}{y^{2}}\right)\left[4 \operatorname{Li}_{4}(-y / x)+4 \mathrm{Li}_{4}(-y)+(3 X-2 Y+i \pi) \operatorname{Li}_{3}(-y / x)\right. \\
& -(X+2 Y+3 i \pi) \operatorname{Li}_{3}(-y)+\left((X-Y)^{2}+\pi^{2}\right) \operatorname{Li}_{2}(-y / x) \\
& +\left((Y+i \pi)^{2}+\pi^{2}\right) \operatorname{Li}_{2}(-y)+\frac{1}{8} X^{2}(X-2 Y)^{2} \\
& \left.-i \frac{\pi}{6} X\left((X+i \pi)^{2}-3 X Y\right)\right] \\
& -\frac{1}{2}\left(1+6 \frac{x}{y^{2}}\right)\left[\operatorname{Li}_{3}(-x)-\zeta_{3}-(X+i \pi)\left(\operatorname{Li}_{2}(-x)-\frac{\pi^{2}}{6}\right)\right. \\
& \left.-\frac{1}{6} X\left(X^{2}+4 \pi^{2}\right)+\frac{1}{2}(X-2 Y-i \pi)\left((X+i \pi)^{2}+\pi^{2}\right)\right] \\
& -\frac{1}{12}\left(5-2 \frac{x}{y}\right)(X+i \pi)\left((X+i \pi)^{2}+3 \pi^{2}\right)+(X-Y)\left((X+i \pi)^{2}+\pi^{2}\right) \\
& +\pi^{2}(X+i \pi)+\frac{1}{8}\left(14 \frac{x-1}{y}-8 y+9 y^{2}\right)\left((X-Y)^{2}+\pi^{2}\right) \\
& +\frac{1}{8}\left(14 \frac{1-x}{y}-8 \frac{y}{x}+9 \frac{y^{2}}{x^{2}}\right)\left((Y+i \pi)^{2}+\pi^{2}\right) \\
& +\frac{1}{8}\left(38 \frac{x}{y^{2}}-13\right)\left((X+i \pi)^{2}+\pi^{2}\right) \\
& -\frac{\pi^{2}}{6}-\frac{9}{4}\left[\left(y+2 \frac{x}{y}\right)(X-Y)-\left(\frac{y}{x}+\frac{2}{y}\right)(Y+i \pi)\right]+\frac{1}{2}, \\
& G_{+--+}^{\mathrm{L}}=2 \frac{1+y^{2}}{x^{2}}\left[\operatorname{Li}_{4}(-y)-\zeta_{4}-\frac{1}{2}(Y+i \pi)\left(\operatorname{Li}_{3}(-y)-\zeta_{3}\right)\right. \\
& \left.+\frac{\pi^{2}}{6}\left(\operatorname{Li}_{2}(-y)-\frac{\pi^{2}}{6}-\frac{1}{2} Y^{2}\right)-\frac{1}{48} Y^{4}-\frac{1}{24}(Y+i \pi)^{2}\left((Y+i \pi)^{2}+\pi^{2}\right)\right] \\
& -2 \frac{3(1-y)^{2}-2 y^{2}}{x^{2}}[
\end{aligned}
$$




$$
\begin{aligned}
\operatorname{Li}_{4}(-x / y)+\mathrm{Li}_{4}(-x)+\mathrm{Li}_{4}(-y)+(X-Y)\left(\mathrm{Li}_{3}(-y)-\zeta_{3}\right) & \left.\pi^{2}\left(\mathrm{Li}_{2}(-y)-X Y-\frac{1}{2} Y^{2}\right)+\frac{1}{4} X^{2} Y^{2}-\frac{1}{6} X Y^{3}-\frac{7}{180} \pi^{4}\right] \\
+ & \frac{2}{3}\left(8-\frac{1}{y}+\frac{30}{x}\right)\left[\operatorname{Li}_{3}(-x / y)-\zeta_{3}-(X-Y)\left(\mathrm{Li}_{2}(-x / y)-\frac{\pi^{2}}{6}\right)\right. \\
& \left.+\frac{1}{2} Y\left((X-Y)^{2}+\pi^{2}\right)\right] \\
+ & \frac{1}{6}\left(4 \frac{x}{y}+27+42 \frac{y}{x}+4 \frac{y^{2}}{x^{2}}\right)\left[\operatorname{Li}_{3}(-y)-\zeta_{3}-(Y+i \pi)\left(\operatorname{Li}_{2}(-y)-\frac{\pi^{2}}{6}\right)\right. \\
\left.-\frac{1}{3} Y\left(Y^{2}+\frac{3}{2} i \pi Y+\pi^{2}\right)\right] & \frac{1}{12}\left(3-2 \frac{y}{x}-12 \frac{y}{x^{2}}\right)(Y+i \pi)\left((Y+i \pi)^{2}+\pi^{2}\right) \\
+ & \frac{x}{3 y}(Y+i \pi)\left((X-Y)^{2}+\pi^{2}\right)+2\left(1+2 \frac{y}{x}\right)\left(\zeta_{3}-\frac{\pi^{2}}{6}(X-Y)\right) \\
+ & \frac{1}{24}\left(\frac{x^{2}}{y^{2}}-24 \frac{x}{y}+44-\frac{8}{x y^{2}}\right)\left((X+i \pi)^{2}+\pi^{2}\right) \\
- & \frac{1}{24}\left(15-\frac{14}{x}-48 \frac{y}{x^{2}}\right)\left((Y+i \pi)^{2}+\pi^{2}\right) \\
+ & \frac{1}{24}\left(\frac{8}{x}+60-24 x+27 x^{2}\right)\left((X-Y)^{2}+\pi^{2}\right)+\frac{4}{9} \frac{\pi^{2}}{x} \\
+ & \frac{2-54 y-27 x^{2}}{12 y}\left(-\frac{1}{x}(Y+i \pi)+(X-Y)\right)
\end{aligned}
$$

where again the variables appearing in the amplitudes are defined in eq. (3.15).

In comparison with the one-loop amplitudes for $g \gamma \rightarrow g \gamma$, the two-loop amplitudes are more singular in the forward limit $u \rightarrow 0$ by one power of $s / u$, due to the exchange of a pair of gluons in the $u$-channel. The power-enhanced terms are only found in the leading color functions $G^{\mathrm{L}}$, and only in the helicity configurations for which the helicity of the incoming gluon does not flip as it scatters (in the conventional helicity labeling, not the all-outgoing one used here). The helicity of the photon may or may not flip in such terms. Explicitly, the power-enhanced terms are

$$
\begin{aligned}
& G_{++++}^{\mathrm{L}} \sim 0, \\
& G_{-+++}^{\mathrm{L}} \sim 2 i \pi \frac{s}{u}, \\
& G_{+-++}^{\mathrm{L}} \sim 0, \\
& G_{-+++}^{\mathrm{L}} \sim-\frac{4}{9}\left(\pi^{2}+3\right) i \pi \frac{s}{u}, \\
& G_{-+-+}^{\mathrm{L}} \sim 0,
\end{aligned}
$$




$$
G_{+--+}^{\mathrm{L}} \sim-\frac{4}{9}\left(\pi^{2}+3\right) i \pi \frac{s}{u} .
$$

These terms represent the beginning of the Reggeization of the $g \gamma \rightarrow g \gamma$ amplitude in perturbation theory; the large $\operatorname{logs}$ of $\ln (-s / u)$ that will arise at subsequent orders in $\alpha_{s}$ could be resummed using BFKL techniques 40 .

\section{Checks on results}

We performed a number of consistency checks on the amplitudes to ensure their reliability:

1. As a check of gauge invariance, we verified that the amplitudes vanish when a gluon or photon polarization vector is replaced with a longitudinal one.

2. The agreement of our explicitly computed infrared divergences with the expected form provides a stringent check on the amplitudes. Since the integrals generally contain both divergent and finite terms, this also provides an indirect verification that the leading color finite remainders have been assembled correctly.

3. Using Supersymmetry Ward Identities [41], we evaluated the identical helicity case 34 by relating it to the already known identical helicity four-gluon amplitudes [10]. Since the integration was done by a completely different technique, the agreement between the two independent ways of evaluating this amplitude provides an additional stringent check of the programs and integration methods used to obtain the general helicity cases.

4. We compared the results for $g g \rightarrow g g$ obtained using our methods and computer programs [35] to those of ref. [13]. The interference of the two-loop $g g \rightarrow g g$ helicity amplitudes with the tree amplitudes, after summing over all external helicities and colors and accounting for the different scheme used (HV vs. CDR), is in complete agreement with the calculation using conventional dimensional regularization $[13]$.

\section{Conclusions}

In this paper we presented the two-loop matrix elements for two gluons to scatter into two photons. Due to the large gluon-gluon luminosity in the $x$ range relevant for the LHC Higgs search, these two-loop contributions should be competitive in size with the 
existing next-to-leading order corrections to the $q \bar{q} \rightarrow \gamma \gamma$ subprocess [6]. The matrix el-

ements presented here, together with the associated real emission contributions, namely the one-loop amplitudes for $g g \rightarrow \gamma \gamma g$ [24, 25], will feed into improved estimates of the QCD background to Higgs production at the LHC, when the Higgs decays into two photons [8].

\section{Acknowledgments}

We thank Adrian Ghinculov and Carl Schmidt for useful conversations.

\section{References}

[1] G. Degrassi, hep-ph/0102137;

J. Erler, hep-ph/0102143.

[2] M. Carena, H.E. Haber, S. Heinemeyer, W. Hollik, C.E. Wagner and G. Weiglein, Nucl. Phys. B 580, 29 (2000) hep-ph/0001002;

J.R. Espinosa and R. Zhang, Nucl. Phys. B 586, 3 (2000) hep-ph/0003246.

[3] R. Barate et al. [ALEPH Collaboration], Phys. Lett. B 495, 1 (2000) hep-ex/0011045;

P. Abreu et al. [DELPHI Collaboration], Phys. Lett. B 499, 23 (2001) hep-ex/0102036;

M. Acciarri et al. [L3 Collaboration], Phys. Lett. B 508, 225 (2001) hep-ex/0012019.

G. Abbiendi et al. [OPAL Collaboration], Phys. Lett. B 499, 38 (2001) hep-ex/0101014.

[4] J. R. Ellis, M. K. Gaillard and D. V. Nanopoulos, Nucl. Phys. B 106, 292 (1976);

M. A. Shifman, A. I. Vainshtein, M. B. Voloshin and V. I. Zakharov, Sov. J. Nucl. Phys. 30, 711 (1979) [Yad. Fiz. 30, 1368 (1979)];

J.F. Gunion, P. Kalyniak, M. Soldate and P. Galison, Phys. Rev. D 34, 101 (1986);

J.F. Gunion, G.L. Kane and J. Wudka, Nucl. Phys. B 299, 231 (1988).

[5] R.K. Ellis, I. Hinchliffe, M. Soldate and J.J. van der Bij, Nucl. Phys. B 297, 221 (1988).

[6] P. Aurenche, A. Douiri, R. Baier, M. Fontannaz and D. Schiff, Z. Phys. C 29, 459 (1985);

B. Bailey, J.F. Owens and J. Ohnemus, Phys. Rev. D 46, 2018 (1992);

B. Bailey and J.F. Owens, Phys. Rev. D 47, 2735 (1993);

B. Bailey and D. Graudenz, Phys. Rev. D 49, 1486 (1994) hep-ph/9307368;

C. Balazs, E.L. Berger, S. Mrenna and C.P. Yuan, Phys. Rev. D 57, 6934 (1998) hepph/9712471];

C. Balazs and C.P. Yuan, Phys. Rev. D 59, 114007 (1999) [Erratum-ibid. D 63, 059902 (1999)] hep-ph/9810319. T. Binoth, J.P. Guillet, E. Pilon and M. Werlen, Eur. Phys. J. C 16, 311 (2000) hep-ph/9911340; Phys. Rev. D 63, 114016 (2001) hep-ph/0012191; T. Binoth, hep-ph/0005194. 
[7] L. Ametller, E. Gava, N. Paver and D. Treleani, Phys. Rev. D 32, 1699 (1985);

D.A. Dicus and S.S. Willenbrock, Phys. Rev. D 37, 1801 (1988).

[8] Z. Bern, L. Dixon and C. Schmidt, in preparation.

[9] Z. Bern, J.S. Rozowsky and B. Yan, Phys. Lett. B 401, 273 (1997) hep-ph/9702424;

Z. Bern, L. Dixon, D.C. Dunbar, M. Perelstein and J.S. Rozowsky, Nucl. Phys. B 530, 401 (1998) hep-th/9802162.

[10] Z. Bern, L. Dixon and D.A. Kosower, JHEP 0001, 027 (2000) hep-ph/0001001.

[11] Z. Bern, L. Dixon and A. Ghinculov, Phys. Rev. D 63, 053007 (2001) hep-ph/0010075.

[12] C. Anastasiou, E.W. Glover, C. Oleari and M.E. Tejeda-Yeomans, Nucl. Phys. B 601, 318 (2001) hep-ph/0010212;

C. Anastasiou, E.W. Glover, C. Oleari and M.E. Tejeda-Yeomans, Nucl. Phys. B 601, 341 (2001) hep-ph/0011094;

C. Anastasiou, E.W. Glover, C. Oleari and M.E. Tejeda-Yeomans, Nucl. Phys. B 605, 486 (2001) hep-ph/0101304.

[13] E.W. Glover, C. Oleari and M.E. Tejeda-Yeomans, Nucl. Phys. B 605, 467 (2001).

[14] W.L. van Neerven, Nucl. Phys. B268, 453 (1986);

Z. Bern, L. Dixon, D.C. Dunbar and D.A. Kosower, Nucl. Phys. B425, 217 (1994) hep$\mathrm{ph} / 9403226$

Z. Bern, L. Dixon, D.C. Dunbar and D.A. Kosower, Nucl. Phys. B 435, 59 (1995) hepph/9409265;

Z. Bern, L. Dixon and D.A. Kosower, Ann. Rev. Nucl. Part. Sci. 46, 109 (1996) hep$\mathrm{ph} / 9602280$.

[15] V.A. Smirnov, Phys. Lett. B460, 397 (1999) [hep-ph/9905323].

[16] J.B. Tausk, Phys. Lett. B469, 225 (1999) hep-ph/9909506.

[17] V.A. Smirnov and O.L. Veretin, Nucl. Phys. B566, 469 (2000) hep-ph/9907385.

[18] C. Anastasiou, T. Gehrmann, C. Oleari, E. Remiddi and J.B. Tausk, Nucl. Phys. B580, 577 (2000) hep-ph/0003261.

[19] T. Gehrmann and E. Remiddi, Nucl. Phys. B580, 485 (2000) hep-ph/9912329.

[20] C. Anastasiou, E.W.N. Glover and C. Oleari, Nucl. Phys. B565, 445 (2000) hepph/9907523;

C. Anastasiou, E.W.N. Glover and C. Oleari, Nucl. Phys. B575, 416 (2000), err. ibid. B585, 763 (2000) hep-ph/9912251. 
[21] S. Catani, Phys. Lett. B427, 161 (1998) hep-ph/9802439.

[22] W.T. Giele and E.W. Glover, Phys. Rev. D 46, 1980 (1992).

[23] Z. Kunszt, A. Signer and Z. Trócsányi, Nucl. Phys. B 420, 550 (1994) hep-ph/9401294.

[24] Z. Bern, L. Dixon and D.A. Kosower, Phys. Rev. Lett. 70, 2677 (1993) hep-ph/9302280.

[25] D. de Florian and Z. Kunszt, Phys. Lett. B 460, 184 (1999) hep-ph/9905283;

C. Balazs, P. Nadolsky, C. Schmidt and C.P. Yuan, Phys. Lett. B 489, 157 (2000) hep-ph/9905551.

[26] See e.g., W.T. Giele, E.W. Glover and D.A. Kosower, Nucl. Phys. B 403, 633 (1993) hep-ph/9302225;

S. Frixione, Z. Kunszt and A. Signer, Nucl. Phys. B 467, 399 (1996) hep-ph/9512328;

S. Catani and M.H. Seymour, Phys. Lett. B 378, 287 (1996) hep-ph/9602277; Nucl. Phys. B 485, 291 (1997) [Erratum-ibid. B 510, 503 (1997)] hep-ph/9605323].

[27] G. 't Hooft and M. Veltman, Nucl. Phys. B44, 189 (1972).

[28] Z. Bern and D.A. Kosower, Nucl. Phys. B379, 451 (1992). Z. Kunszt, A. Signer and Z. Trócsányi, Nucl. Phys. B411, 397 (1994) hep-ph/9305239. S. Catani, M.H. Seymour and Z. Trócsányi, Phys. Rev. D55, 6819 (1997) hep-ph/9610553.

[29] Z. Bern and A.G. Morgan, Nucl. Phys. B 467, 479 (1996) hep-ph/9511336.

[30] F.A. Berends, R. Kleiss, P. De Causmaecker, R. Gastmans and T.T. Wu, Phys. Lett. B 103, 124 (1981);

P. De Causmaecker, R. Gastmans, W. Troost and T.T. Wu, Phys. Lett. B 105, 215 (1981);

Z. Xu, D. Zhang and L. Chang, Nucl. Phys. B 291, 392 (1987).

[31] M.L. Mangano and S.J. Parke, Phys. Rept. 200, 301 (1991);

L. Dixon, in Proceedings of Theoretical Advanced Study Institute in Elementary Particle Physics (TASI 95), ed. D.E. Soper [hep-ph/9601359].

[32] D.A. Kosower, Phys. Lett. B 254, 439 (1991).

[33] Z. Bern, L. Dixon and D.A. Kosower, Phys. Lett. B 302, 299 (1993) [Erratum-ibid. B 318, 649 (1993)] hep-ph/9212308;

Z. Bern, L. Dixon and D.A. Kosower, Nucl. Phys. B 412, 751 (1994) hep-ph/9306240].

[34] Z. Bern, A. DeFreitas, L. Dixon and H.L. Wong, to appear.

[35] Z. Bern, A. De Freitas, L. Dixon and A. Ghinculov, in preparation.

[36] See e.g. K.S. Kölbig, SIAM J. Math. Anal. 17, 1232 (1986). 
[37] L. Lewin, Dilogarithms and Associated Functions (Macdonald, 1958).

[38] K.S. Kölbig, J.A. Mignaco and E. Remiddi, B.I.T. 10, 38 (1970).

[39] Z. Bern, A. De Freitas, L. Dixon, A. Ghinculov and H.L. Wong, preprint SLAC-PUB8974, UCLA/01/TEP/18, hep-ph/0109079

[40] E. A. Kuraev, L. N. Lipatov and V. S. Fadin, Sov. Phys. JETP 44, 443 (1976) [Zh. Eksp. Teor. Fiz. 71, 840 (1976)];

E.A. Kuraev, L.N. Lipatov and V.S. Fadin, Sov. Phys. JETP 45, 199 (1977) [Zh. Eksp. Teor. Fiz. 72, 377 (1977)];

I.I. Balitsky and L.N. Lipatov, Sov. J. Nucl. Phys. 28, 822 (1978) [Yad. Fiz. 28, 1597 (1978)].

[41] M.T. Grisaru, H.N. Pendleton and P. van Nieuwenhuizen, Phys. Rev. D 15, 996 (1977); M.T. Grisaru and H.N. Pendleton, Nucl. Phys. B 124, 81 (1977);

S.J. Parke and T.R. Taylor, Phys. Lett. B 157, 81 (1985), err. ibid. 174B, 465 (1985). 